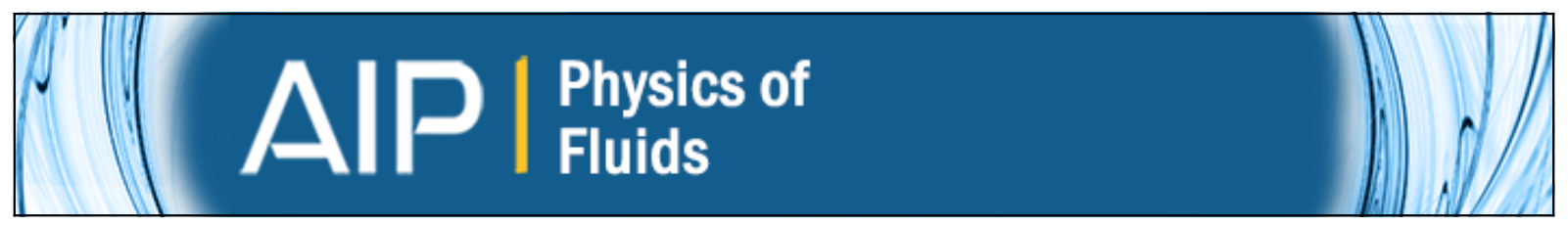

\title{
Dynamics of nanoscale jet formation and impingement on flat surfaces
}

Sohail Murad and Ishwar K. Puri

Citation: Physics of Fluids (1994-present) 19, 128102 (2007); doi: 10.1063/1.2773999

View online: http://dx.doi.org/10.1063/1.2773999

View Table of Contents: http://scitation.aip.org/content/aip/journal/pof2/19/12?ver=pdfcov

Published by the AIP Publishing

Articles you may be interested in

Interaction of a synthetic jet with the flow over a low aspect ratio cylinder

Phys. Fluids 25, 064104 (2013); 10.1063/1.4811710

Surface properties for rarefied circular jet impingement on a flat plate

Phys. Fluids 23, 027102 (2011); 10.1063/1.3549934

Global and local instability of flow focusing: The influence of the geometry

Phys. Fluids 22, 064105 (2010); 10.1063/1.3450321

Dynamics of liquid jets and threads under the action of radial electric fields: Microthread formation and touchdown singularities

Phys. Fluids 21, 032109 (2009); 10.1063/1.3097888

Simulations of starting gas jets at low Mach numbers

Phys. Fluids 17, 038105 (2005); 10.1063/1.1858533

\section{AlP Re-register for Table of Content Alerts}




\title{
Dynamics of nanoscale jet formation and impingement on flat surfaces
}

\author{
Sohail Murad and Ishwar K. Puria) \\ Department of Chemical Engineering, University of Illinois at Chicago, Chicago, Illinois 60607, USA \\ and Department of Engineering Science and Mechanics, 223 Norris Hall, \\ Virginia Polytechnic Institute and State University, Blacksburg, Virginia 24061, USA
}

(Received 17 July 2007; accepted 27 July 2007; published online 7 December 2007)

\begin{abstract}
Molecular-dynamics simulations are used to investigate the formation of water nanojets. The fluid is forced through a nano-orifice to establish a nanojet, which then impinges on a flat surface. The simulations show that to produce jets in the $1 \mathrm{~nm}$ diameter range, the orifice surface must be hydrophobic, otherwise the nanojet kinetic energy/inertia may never be able to overcome the attractive forces of the surface to form a jet. In addition, for the nanojet to form a stable liquid film on the surface of impingement, the surface cannot be either hydrophobic or too hydrophilic. Finally the stability/formation of the nanojet is not sensitive to the orifice surface temperature. The same physical laws that govern flows at the micro- and macroscales adequately describe nanojet flows in the absence of strong interfacial forces. (c) 2007 American Institute of Physics.
\end{abstract}

[DOI: $10.1063 / 1.2773999$ ]

We report investigations of the dynamics of nanojets produced by forcing water through a $1 \mathrm{~nm}$ orifice, and its subsequent impingement on a flat surface, to determine how interfacial conditions modify their behavior. Simulations of flow involving high kinetic energy nanojets must be capable of resolving length and time scales that are as small as $10^{-11} \mathrm{~m}$ and $10^{-12} \mathrm{~s},{ }^{1}$ which we accomplish through molecular-dynamics (MD) simulations. The method has been used to probe nanoscale liquid behavior at interfaces. ${ }^{1-6}$ The placement of boundaries and how these interfaces behave has a significant influence on nanoscale fluid flows compared to larger scale flows due to their larger surface-to-volume ratios. ${ }^{1}$ For instance, the transport of polar water molecules inside a carbon nanotube ${ }^{1}$ can be significantly enhanced by charging its wall to induce hydrophilic interactions. ${ }^{3}$ In the absence of such effects, a nanoscale flow follows the regular continuum scale physical relations, ${ }^{4}$ even for nanoscale segments containing only ten molecules in each spatial direction. ${ }^{5}$ For water, the viscosity $\mu \approx 10^{-3} \mathrm{~Pa}$ s and density $\rho=10^{3} \mathrm{~kg} \mathrm{~m}^{-3}$, i.e., the viscous force $\mu^{2} / \rho \approx 10^{-9} \mathrm{~N}{ }^{7}$ Clearly, relatively small interfacial forces of the order of $10^{-9} \mathrm{~N}$ can significantly influence nanoscale flows.

A detailed description of our MD simulation methodology is available elsewhere. ${ }^{8,9}$ A Gaussian thermostat maintains constant temperature ${ }^{10}$ and the molecules are assumed to have an initial Gaussian velocity distribution corresponding to the system temperature. A fifth-order Gear predictorcorrector algorithm for translational motion and a fourthorder predictor-corrector algorithm for rotational motion are used with the quaternion method. ${ }^{10,11}$ Site-site interactions are based on the potential model $u_{i j}=4 \varepsilon_{i j}\left[\left(\sigma_{i j} / r_{i j}\right)^{12}\right.$ $\left.-\left(\sigma_{i j} / r_{i j}\right)^{6}\right]+\left(q_{i} q_{j}\right) / r_{i j}$. The potential parameters $\sigma$ and $\varepsilon$, and charges $q$, obtained from the literature, have been shown to closely represent experimental geometries, energies of ionwater complexes, and energies of solvation. ${ }^{3}$ In the equation,

${ }^{a)}$ Electronic mail: ikpuri@vt.edu denotes the scalar distance between sites $i$ and $j$, and are the LJ interaction parameters, and $q_{i}$ and $q_{j}$ represent the Coulombic charges on sites $i$ and $j$, although not all sites have charges. Lorentz-Berthelot mixing rules are used for cross interactions ${ }^{10}$ and the reaction field method is used to model long-range interactions. ${ }^{12,13}$ Water is modeled using the SPC potential ${ }^{14}$ and the membrane atoms are modeled either as LJ sites to replicate hydrophobic walls or with additional charges, both positive and negative to maintain overall charge neutrality, that render them hydrophilic. We alternated the sign of the local charge $(+q,-q)$ between adjacent atoms to mimic a polar surface.

The simulations mimic a hypothetical nanoscale laboratory experiment in which we consider 1503 molecules of water that are initially contained in two connected reservoirs. The composite reservoir is restricted by movable walls that contain five carbon layers, each containing 119 atoms arrayed in an fcc lattice, as shown in a side $(y-z)$ view of the simulation domain in Fig. 1(a). Both reservoirs enclose a fully evacuated cavity that, acting like the plunger of a syringe, ejects fluid through the orifice. As the reservoir walls are squeezed, a nanojet forms and flows through the orifice into the cavity. The system temperature is $343 \mathrm{~K}$. Our simulations have shown that varying the wall temperature between 298 and $343 \mathrm{~K}$ has only a marginal effect on the nanojet ejection rate. Periodic boundary conditions are employed. We are able to readily simulate nanojets with an initial diameter of $\approx 1 \mathrm{~nm}$.

The simulations are conducted in dimensionless units $\left(L_{x}{ }^{*}, L_{y}{ }^{*}, L_{z}{ }^{*}\right)=(39.3,19.65,19.65)$. Each simulation typically runs for more than $6 \times 10^{5}$ time steps, with each step being $1.7591 \times 10^{-16} \mathrm{~s}$. The molecules constituting the movable membranes are tethered using a simple harmonic potential that represents the thermal fluctuations of the wall molecules. As these tethering sites are moved while they continue to undergo thermal fluctuations, the movable membranes are also dragged toward the system boundaries with 


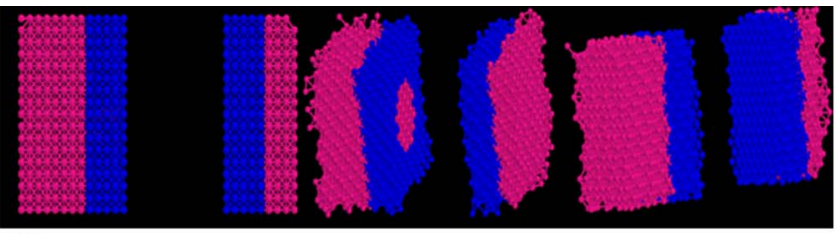

(a)

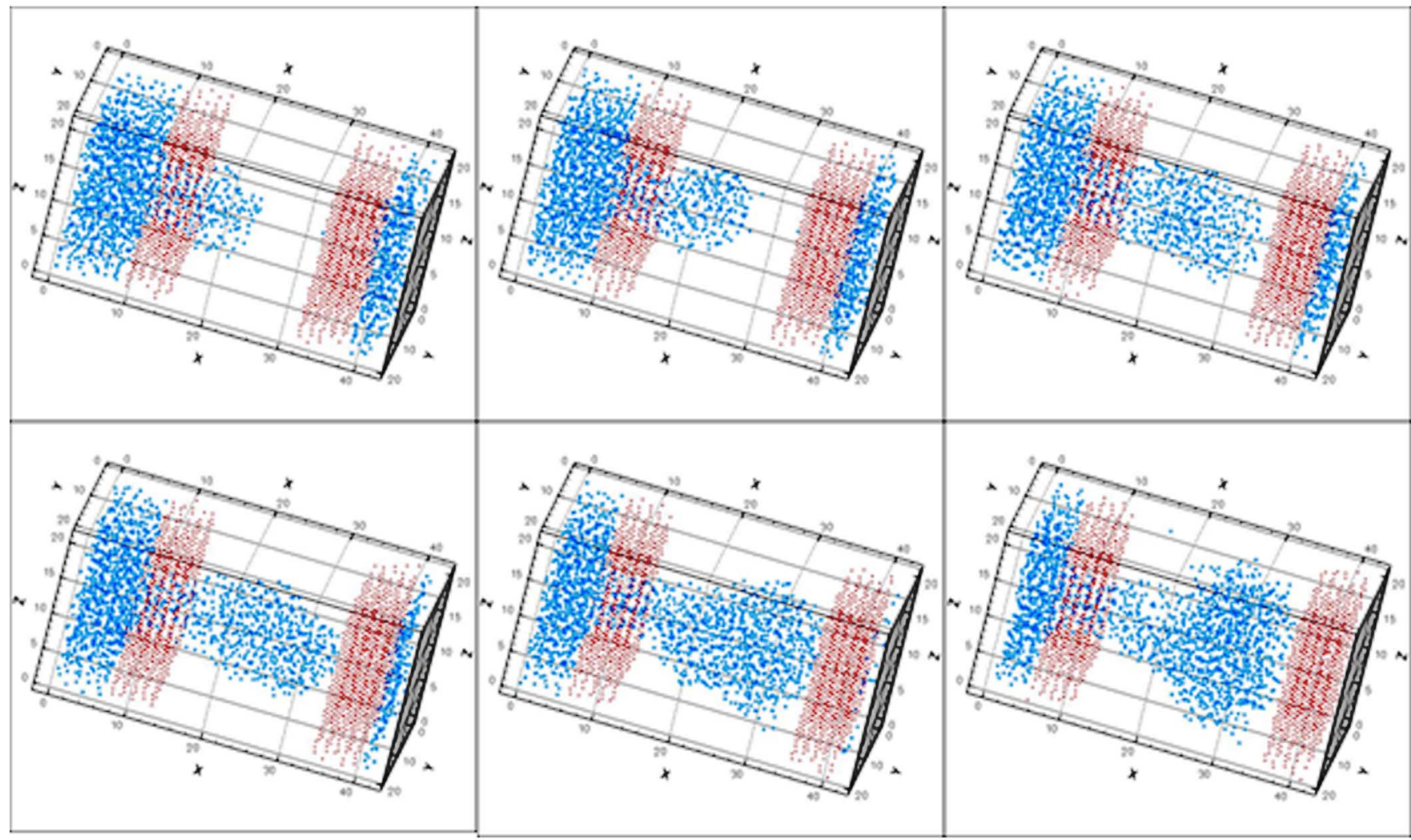

(b)
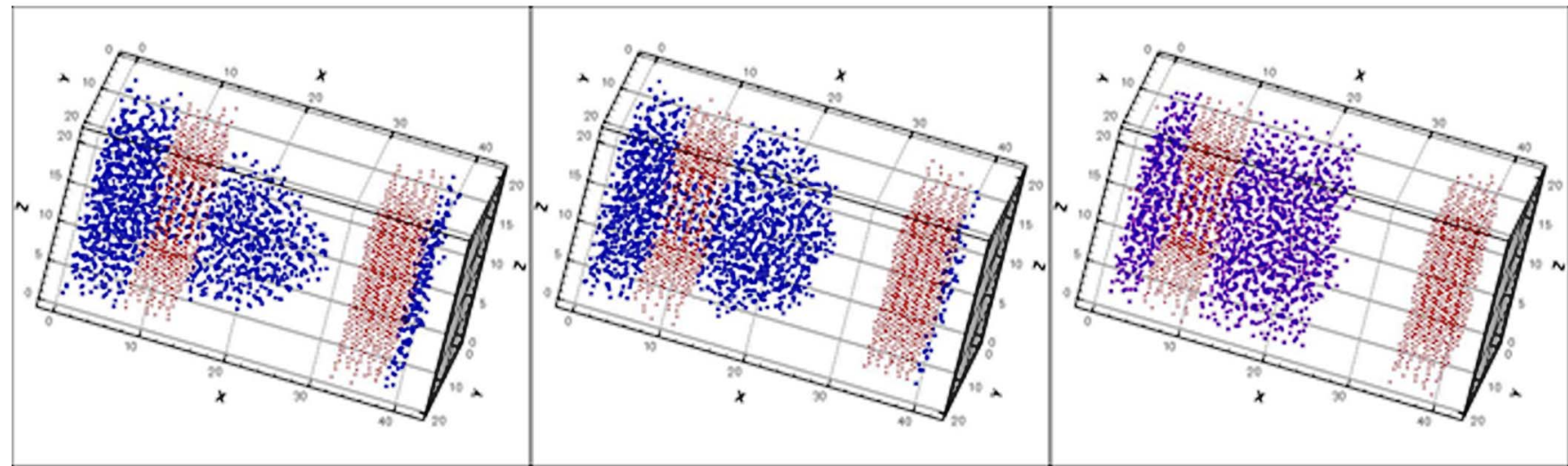

(c)

FIG. 1. (Color online) Schematic illustration of the simulation system. One wall has an orifice through which the nanojet emerges while the opposite wall serves as a stagnation surface. The simulation domain is a parallelepiped with the two movable membranes initially placed at $x=L_{x} / 4$ and $3 L_{x} / 4$. Including the two water reservoirs at each periphery, the end-to-end dimensions of the simulation box are $\left(L_{x}, L_{y}, L_{z}\right)=(7.46,3.73,3.73) \mathrm{nm}$. (b) Nanojet formation and stagnation dynamics for hydrophobic interfaces at the orifice and stagnation wall. The average nanojet velocity $U_{\text {av }, o} \approx 140 \mathrm{~m} / \mathrm{s}$. The nanojet fluid is colored blue and the membrane molecules are red. Results are presented from 100000 to 600000 time steps at intervals of 100000 steps. (c) Influence of a hydrophilic orifice wall on nanojet formation and propagation from 300000 to 600000 time steps at intervals of 150000 steps. Here, each of the orifice wall atoms is provided with an alternating charge $\left|q^{*}\right|=1.25\left(q^{*}=10.45=1 e\right.$ charge $)$. 


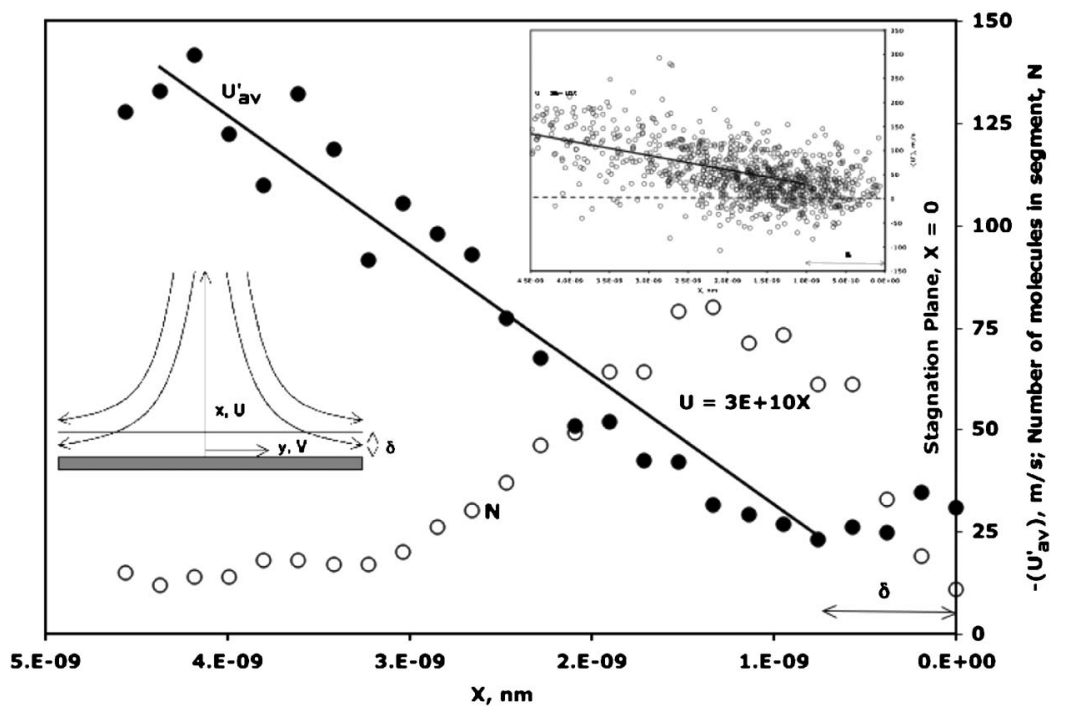

FIG. 2. The number of water molecules in successive $0.19 \mathrm{~nm}$ strips and the averaged nanojet velocity profile through these segments after $6 \times 10^{5}$ time steps for the simulations of Fig. 1(a). The corresponding spatial velocity distribution is presented in an inset. constant velocities of up to $8.6 \mathrm{~m} / \mathrm{s}$ and produce nanojet ejection velocities $U$ of up to $\approx 150 \mathrm{~m} / \mathrm{s}$. Molecules within a flowing water cluster interact due to both Coulombic and van der Waals forces. Water molecules interact with the walls through van der Waals forces for hydrophobic surfaces, and also with surface Coulombic forces when the interfaces are hydrophilic. In general, larger membrane displacement (squeezing) rates result in nanojets that have more uniform axial velocities. Increasing the liquid pressure speeds up jet formation without influencing the jet integrity. Figure 1(b) shows the development of a water nanojet emerging through a hydrophobic orifice and its impingement dynamics on a hydrophobic stagnation surface. If the orifice surface is made strongly hydrophilic, the nanojet never emerges from the surface, as shown in Fig. 1(c). This is because the kinetic energy of the jet is overwhelmed by the strong attraction between the wall and the surface and is unable to break away from it. This also explains the reversal in the motion of the water molecules that is caused by the electrostatic field.

We segment the simulation domain into $\Delta x^{*}=1$ axial strips and average the local velocity in each strip. The number of water molecules in each strip and the averaged nanojet velocity profile obtained after $6 \times 10^{5}$ time steps are presented in Fig. 2 (for which $X=0$ at the stagnation plane). Consistent with the behavior of a stagnating flow, the corresponding axial velocity $U^{\prime}$ has increasing upstream values. Only a few molecules accumulate adjacent to the hydrophobic interface as a result of the absence of any strong watermembrane attractive forces. The corresponding spatial velocity distribution is presented in an inset in the figure. At that time, the averaged orifice ejection velocity $U_{\mathrm{av}, o}^{\prime} \approx 140 \mathrm{~m} / \mathrm{s}$ and the axial flow dimension (left to right membrane) $L_{o}$ $\approx 4.5 \mathrm{~nm}$. Since $a=U / L_{o}$, its value is $3.1 \times 10^{10} \mathrm{~s}^{-1}$. Based on an analysis of the Navier-Stokes equations, ${ }^{15}$ a macroscale laminar jet brought to stagnation against a flat plate follows the local relations $U^{\prime}=-a X$ and $V^{\prime}=a Y$. As shown in Fig. 2, the averaged axial velocity profile $U_{\mathrm{av}}^{\prime}=3$ $\times 10^{10} \mathrm{X} \mathrm{m} \mathrm{s}^{-1}$, which is consistent with this continuum relation, and once again shows such applicability in the nanoscale regime. ${ }^{4}$ This relation does not hold in a small viscous layer that contains a relatively large number of molecules and where the velocity has an almost constant small value. Results for other time steps follow similar relations, although the values for $U_{\mathrm{av}, o}^{\prime}$ and $L_{o}$, and therefore $a$, are different.

The utility of comparing a continuum approach with the results of nanoscale MD fluid simulations has been previously stated. For instance, the hydrodynamic forces simulated on a carbon nanotube have been found to be in good agreement with the macroscopic Stokes-Oseen solution for the flow past an array of circular cylinders. ${ }^{19}$ The implication of such studies is that nanoscale fluid flow problems do not always occur below the smallest continuum scale. Since a point function, such as density, based on a continuum approach is an average property, it could be adequately described provided the nanoscale volume of interest contains a sufficient number of molecules.

Our results can be also contrasted with other recent MD simulations of nanojet formation and breakup. ${ }^{16-18}$ They do not show the significant deviations from continuum theories that have been previously reported and attributed to thermal fluctuations. ${ }^{16,17}$ We believe this is primarily due to our consideration of a strongly polar fluid (water) that has significant intermolecular interactions (due to the high dipole and hydrogen bonding in water) as compared to the previous studies of nonpolar Lennard-Jones systems. ${ }^{16-18}$ With the much stronger intermolecular interactions in our case, molecules lose memory of their initial or previous velocities much more rapidly after collisions occur, thereby significantly attenuating the role of thermal fluctuations in fluids like water. This is an important effect that must be kept in mind when examining the role of thermal fluctuations in nanoscale systems.

Results for stagnating surfaces that are moderately or strongly hydrophilic (when the orifice wall is hydrophobic) are presented in Fig. 3. The nanojet spreading observed in Fig. 1(b) is enhanced by the presence of the hydrophilic stagnation interface placed downstream of it. The wall enhances the layering of water molecules adjacent to it, as illustrated through the insets in Fig. 3. The region of the jet that is not directly influenced by the hydrophilic interface continues to follow the linear stagnation velocity profile $U^{\prime}=-a X$ until a 


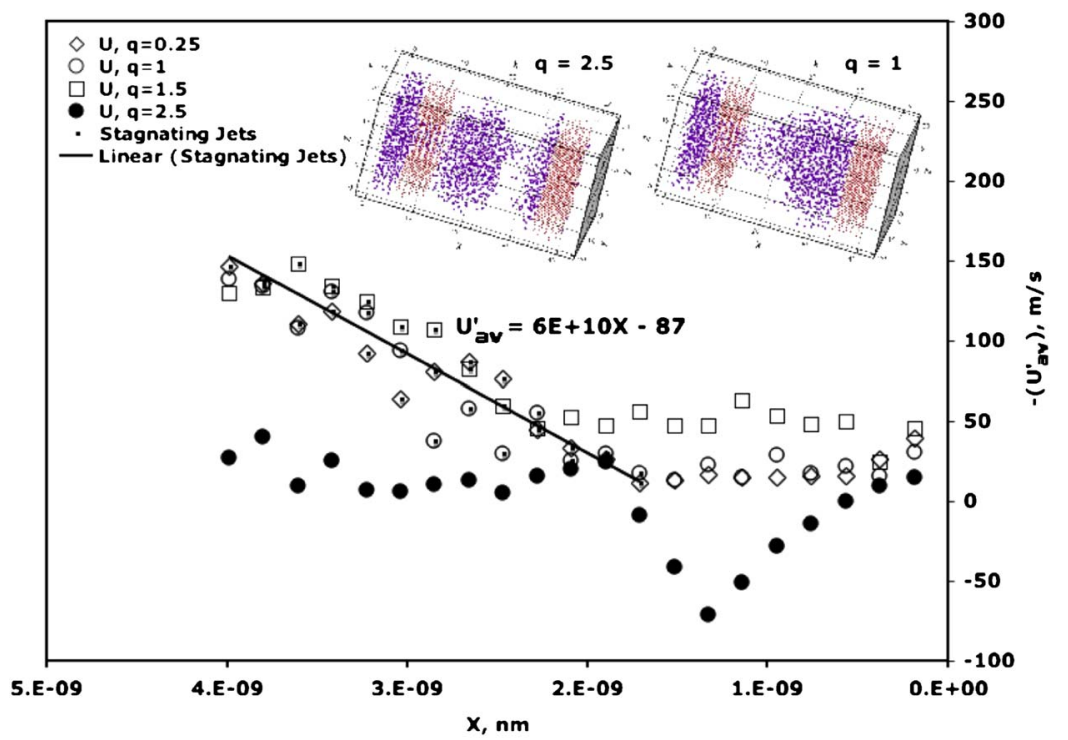

FIG. 3. (Color online) The average nanojet velocity profile for various charges. The insets show the nanojet spatial distributions for two cases, when $|q|=1$ and 2.5 after 600000 time steps. critical charge value. Thereafter, as the surface charge increases, the bulk of the nanojet is repelled toward the orifice wall forming a film on it, in a manner similar to the results presented in Fig. 1(c). The nanojet is then unable to propagate through this film adjacent to the orifice and its ejection velocity is considerably reduced. It is interesting to speculate why the liquid film for strongly hydrophilic surfaces $(q$ $=2.5)$ is in fact thinner and less stable than that for less hydrophilic surfaces $(q=1)$. We conjecture that a highly hydrophilic interface attracts polar water molecules in a manner that is not conducive to optimal hydrogen bonding with a successive layer. Consequently, bonding with the first layer is weakened so that water molecules in outer layers of the film build stronger hydrogen bonds with proximal molecules outside the film to which they are energetically more attracted. In contrast, molecules attracted to hydrophilic walls that induce weaker surface interactions are freer to change their orientation and to form more favorable hydrogen bonds. They can thus establish several adjacent molecular layers in favorably orientated configurations, resulting in thicker films.

In summary, we have shown that the same physical laws that govern flows at the micro- and macroscales also adequately describe nanojet flows in the absence of strong interfacial forces. Introducing a hydrophilic interface adjacent to a nanoscale flow significantly influences it. However, an interfacial effect can be nonlinear, since it influences not only water-interface but also water-water interactions. A strongly hydrophilic interface, for example, inhibits stable surface film formation, because we believe the orientation of the polar water molecules in the film is no longer conducive to optimal hydrogen bonding with a successive layer.
${ }^{1}$ S. Banerjee, S. Murad, and I. K. Puri, "Preferential ion and water intake using charged carbon nanotubes," Chem. Phys. Lett. 434, 292 (2007).

${ }^{2}$ P. A. Thompson and S. Troian, "A general boundary condition for liquid flow at solid surfaces," Nature 389, 360 (1997).

${ }^{3}$ J. Chandrasekhar, D. C. Spellmeyer, and W. L. Jorgensen, "Energy component analysis for dilute aqueous solutions of $\mathrm{Li}[+], \mathrm{Na}[+] \mathrm{F}[-]$, and Cl[-] ions," J. Am. Chem. Soc. 106, 903 (1984).

${ }^{4}$ S. Murad and I. K. Puri, "Nanoscale jet collision and mixing dynamics," Nano Lett. 7, 707 (2007).

${ }^{5}$ J. Koplik and R. Banavar, "Continuum deductions from molecular simulations," Annu. Rev. Fluid Mech. 27, 257 (1995).

${ }^{6}$ M. Gad-el-Hak, "Liquids: The holy grail of microfluidic modeling," Phys. Fluids 17, 100612 (2005).

${ }^{7}$ E. M. Purcell, "Life at low Reynolds number," Am. J. Phys. 45, 3 (1977).

${ }^{8}$ S. Murad and J. Lin, "Molecular modeling of fluid separations using membranes: Effect of molecular forces on mass transfer rates," Chem. Eng. J. 74, 99 (1999).

${ }^{9}$ S. Murad and J. Lin, "Using thin zeolite membranes and external electric fields to separate supercritical aqueous electrolyte solutions," Ind. Eng. Chem. Res. 41, 1076 (2002).

${ }^{10}$ M. P. Allen and D. J. Tildesley, Computer Simulation of Liquids (Clarendon Press, Oxford, 1987).

${ }^{11}$ D. J. Evans and S. Murad, "Singularity free algorithm for molecular dynamics simulation of rigid polyatomics," Mol. Phys. 34, 327 (1977).

${ }^{12}$ I. G. Tironi, R. Sperb, P. E. Smith, and W. F. van Gunsteren, "A generalized reaction field method for molecular dynamics simulations," J. Chem. Phys. 102, 5451 (1995).

${ }^{13}$ H. J. C. Berendson, J. Postma, and W. F. van Gunsteren, in Intermolecular Forces, edited by B. Pullman (Reidel, Dordrecht, 1981).

${ }^{14}$ R. O. Watts, "Monte Carlo studies of liquid water," Mol. Phys. 28, 1069 (1974).

${ }^{15}$ H. Schlichting, Boundary Layer Theory (McGraw-Hill, New York, 1968).

${ }^{16}$ M. Mozeler and U. Landman, Science 289, 1165 (2000).

${ }^{17}$ Y. S. Choi, S. J. Kim, and U. Kim, "Molecular dynamics of unstable motions and capillary instability in liquid nanojets," Phys. Rev. E 73, 016309 (2006).

${ }^{18}$ T. Fang, W. Chang, and S. Liao, "Simulated nanojet ejection process by spreading droplets on a solid surface," J. Phys.: Condens. Matter 15, 8263 (2003).

${ }^{19}$ J. H. Walther, T. Werder, R. L. Jaffe, and P. Koumoutsakos, "Hydrodynamic properties of carbon nanotubes," Phys. Rev. E 69, 062201 (2004). 